\title{
SPECTROSCOPIC OBSERVATIONS OF BE STARS \\ IN THE NEAR INFRARED
}

\author{
R. S. POLIDAN and G. J. PETERS \\ Dept. of Astronomy, University of California, Los Angeles, Calif., U.S.A.
}

\begin{abstract}
The results of near infrared spectroscopic observations of over $120 \mathrm{Be}$ stars are presented. In particular, the $O$ I lines at $\lambda 7774$ and $\lambda 8446 \AA$ and the Ca II triplet $(\lambda 8498 \AA, \lambda 8542 \AA$, and $\lambda 8662 \AA)$ are discussed. The presence of emission at $\mathrm{O}_{\text {I }} \lambda 7774 \AA$ is shown to correlate with Fe II emission. Shell absorption at this line is found to be similar in appearance to that found in the photospheric spectra of supergiants. The O I $\lambda 8446 \AA$ line, the result of a fluorescence with Lyman $\beta$, is briefly discussed in connection with $\mathrm{Be}$ envelope dimensions. The paradox presented by the presence of strong $\mathrm{Ca}$ II triplet emission without $\mathrm{H}$ and $\mathrm{K}$ emission is discussed. The implications of this phenomenon on the structure of Be envelopes and possibly the binary nature of some Be stars is discussed.

Evidence indicating the presence of cool companions to the Be stars HD 218393 and HR 894 is presented.
\end{abstract}

\section{Introduction}

In 1972 we, in collaboration with Dr M. Plavec, initiated a program to study the red and near infrared spectra of Be stars, shell stars, and interacting binary stars. Earlier investigations of Be stars in this region by Hiltner (1947), Slettebak (1951), and Andrillat and Houziaux $(1967,1972)$ revealed that a systematic, quantitative, study of emission line stars in this region could yield important information on the structure of their envelopes and possibly on the relationship between Be stars and interacting binary stars.

\section{Observations}

The observations were made using the Lick Observatory $40 \mathrm{~mm}$ cooled Varo image intensifier in conjunction with the $24-i n .(61 \mathrm{~cm})$ Coude Auxiliary Telescope. A substantial number of observations were also made with the 120 -in. $(305 \mathrm{~cm})$ telescope through the cooperation of Dr M. Plavec. Over 500 near infrared plates of over $120 \mathrm{Be}$ stars have been obtained during the three years of observation. The spectrograms in the region $\lambda \lambda 7500-8200 \AA$ were taken at dispersions of $34 \AA \mathrm{mm}^{-1}$ (85\% of the total) and $12 \AA \mathrm{mm}^{-1}$. In the region of the higher order Paschen lines $(\lambda \lambda 8200-8700 \AA)$, ninety percent of the stars were observed at $23 \AA \mathrm{mm}^{-1}$ and 10 percent at $34 \AA^{\mathrm{mm}}{ }^{-1}$. All spectra were widened to $0.6 \mathrm{~mm}$ at the plate. The characteristics of the Lick Observatory Varo tube have been described by Zappala (1971). The spatial resolution of the tube is comparable with that of a I-N plate. However, the Varo tube's high degree of freedom from small scale irregularities combined with the high contrast and low noise of III-aJ plates produce a spectrum of considerably higher quality than is possible from I-N plates. 


\section{Data}

The near infrared region $(\lambda \lambda 7500-8800 \AA)$ of Be stars is dominated by the Paschen lines $P 12$ and higher, the neutral oxygen lines at $\lambda 7774 \AA$ and $\lambda 8446 \AA$, and by the infrared calcium triplet ( $\lambda 8498 \AA, 8542 \AA$, and $8662 \AA)$. The Fe II emission line at $\lambda 7712 \AA$ is seen in those stars exhibiting Fe II emission in the blue/violet spectral region. This region is devoid of metallic shell lines except for the

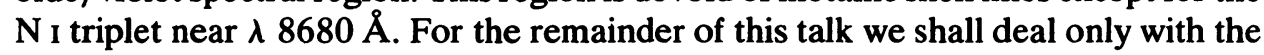
results for the two oxygen lines and for the calcium triplet.

\subsection{O I $\lambda 7774 \AA$}

The neutral oxygen triplet at $\lambda 7774 \AA(\lambda 7772,4$, and 5) arises from the transition between the metastable ${ }^{5} S$ level and the ${ }^{5} P$ level. As a consequence of the metastability of the lower level this feature has been shown to be a useful luminosity indicator in non-emission line stars (see, for example, Osmer, 1972). Our results for Be stars show that twenty percent display emission at O I $\lambda 7774 \AA$ and twenty percent have enhanced (shell) absorption. The remaining sixty percent show only the absorption lines expected from the underlying star's photosphere. Figure 1 shows

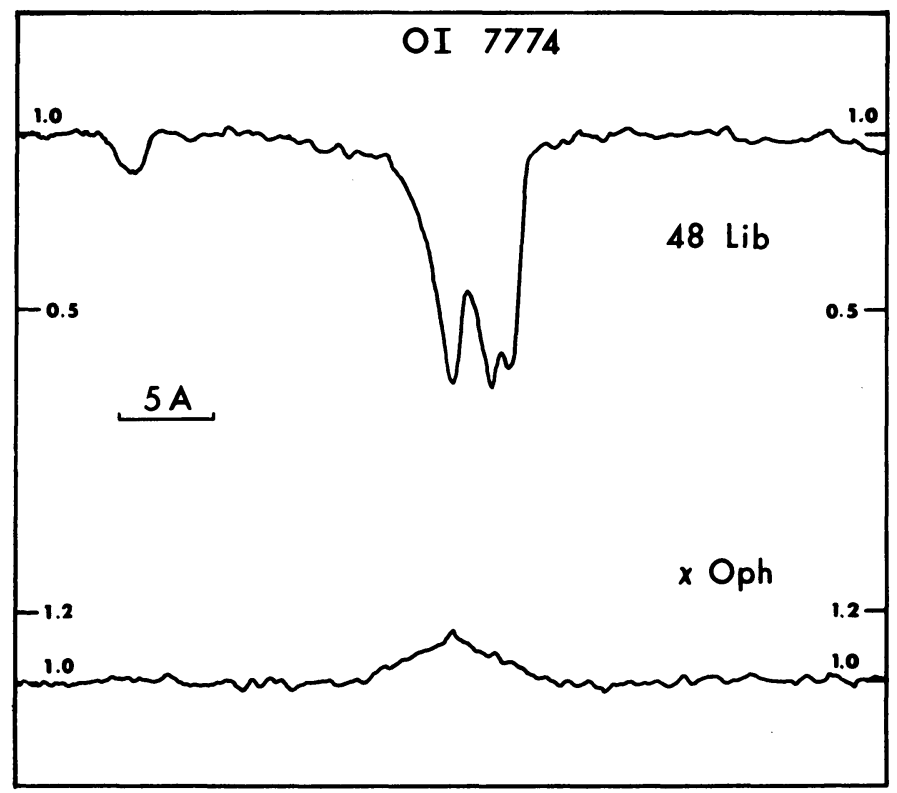

Fig. 1. Intensity tracings of the Or $\lambda 7774 \AA$.region in the shell star $48 \mathrm{Lib}$ and the Be star $\chi$ Oph. Ordinate is in intensity units normalized to the continuum. Original dispersion: $12 \AA \mathrm{mm}^{-1}$.

tracings of the region around $O_{\text {I }} \lambda 7774 \AA$ in the objects showing the strongest emission $(\chi \mathrm{Oph})$ and the strongest shell absorption $(48 \mathrm{Lib})$.

All stars which show $\mathrm{O}_{\mathrm{I}} \lambda 7774 \AA \AA$ emission are observed to have $\mathrm{Fe}$ II emission. However, the reverse is not necessarily true. Objects do exist for which Fe II emission 
is seen without accompanying OI $\lambda 7774 \AA$ emission (e.g. $\beta^{1}$ Mon). Most OI emission objects also display strong $\mathrm{H} \alpha$ emission with little or no structure. A correlation was found to exist between the strength of the $\mathrm{O}_{\mathrm{I}} \lambda 7774 \AA$ emission and the Fe II $\lambda 7712 \AA$ emission line (Figure 2). A correlation also exists between the width of the $\mathrm{O}$ I emission and the width of the $\mathrm{Fe}$ II feature. Both also correlate with $v \sin i$ of the underlying star.

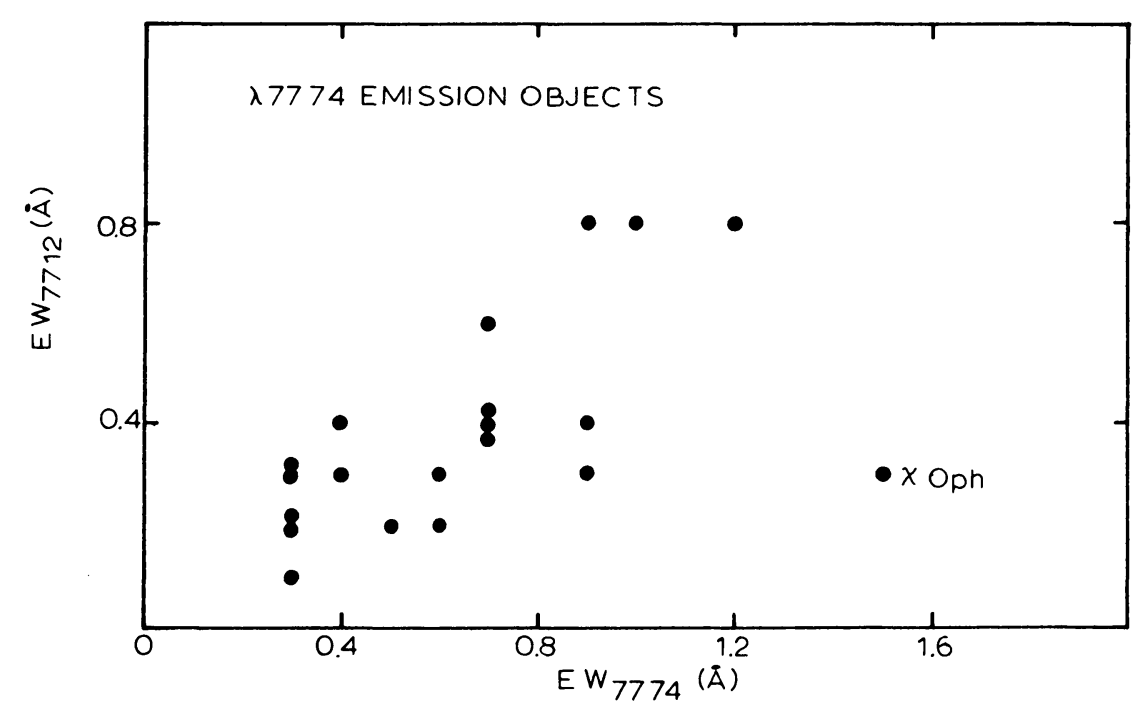

Fig. 2. Observed correlation between the equivalent widths of Fe II $\lambda 7.712 \AA$ and $O_{1} \lambda 7774 \AA$.

The tentative conclusion is that the region giving rise to the $\mathrm{O}_{1}$ emission is the same as that giving rise to the $\mathrm{Fe}$ II emission.

Because of the strong metastability of the lower level the O I $\lambda 7774 \AA$ line is a useful indicator of a shell star. Stars exhibiting only a weak metallic shell in the blue show significant enhancement of this line (e.g. $o$ And). In the case of stars with strong metallic shells (e.g. $48 \mathrm{Lib}$, Figure 1), the $\mathrm{O}_{\mathrm{I}}$ triplet is seen in absorption stronger than is observed in the most extreme supergiants. In general the appearance of the line is similar to what is seen in normal giants and supergiants. This suggests that in some cases the physical characteristics and structure of a shell star envelope may be quite similar to the atmosphere of a giant or supergiant star.

\subsection{O I $\lambda 8446 \AA$}

Emission in this line was found in $75 \%$ of the Be stars surveyed. The explanation for the high percentage of emission was pointed out by Bowen (1947): the line is the result of a fluorescence with $L \beta$. Kitchin and Meadows (1970) investigated the expected correlation between the emission strengths of $\mathrm{H} \alpha$ and $\mathrm{O}_{\mathrm{I}} \lambda 8446 \AA$. Our results support their conclusion that the correlation does exist. However, we have found a few exceptions to the relation (e.g. $31 \mathrm{Peg}$ ). 
Kitchin (1970) has used the $\mathrm{O}_{\text {I }} \lambda 8446 \AA$ line to obtain dimensions for the emitting regions around $\mathrm{Be}$ stars. His model is based on the assumption that the line is optically thin. Our observations show that in all but the sharpest lined stars ( $v \sin i>150 \mathrm{~km} \mathrm{~s}^{-1}$ ) a strong reversal is present at the line center. This is inconsistent with the assumption that the line is optically thin. Therefore, envelope dimensions based on an optically thin O I $\lambda 8446 \AA$ should be regarded with caution.

Figure 3 shows tracings of the Paschen region $(\lambda \lambda 8400-8575 \AA)$ in the Be star $\chi$ Oph and the shell star $48 \mathrm{Lib}$.

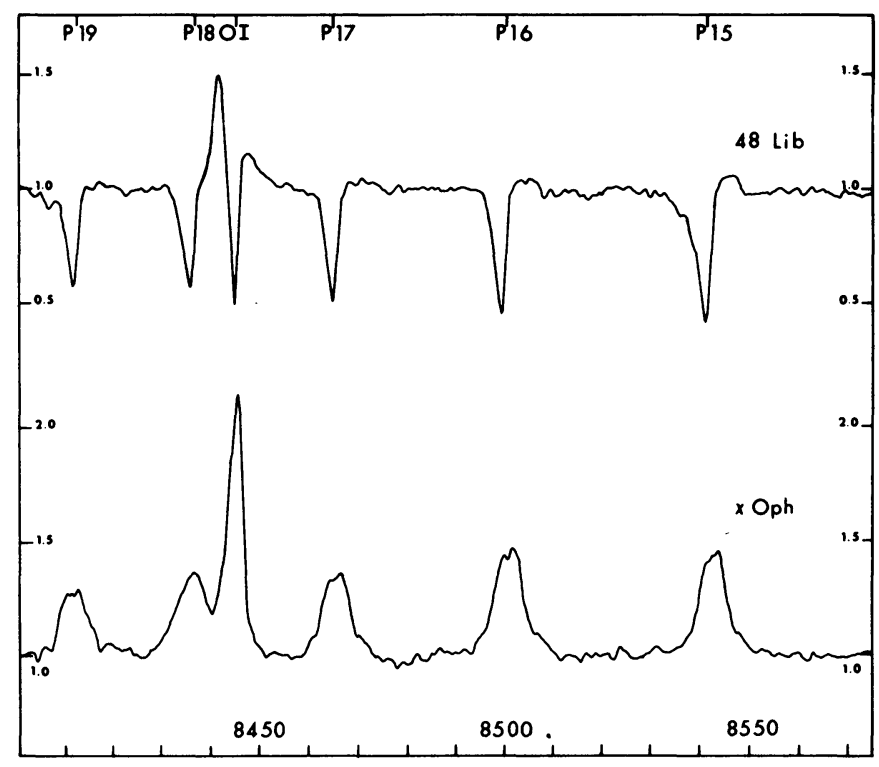

Fig. 3. Spectra of the shell star $48 \mathrm{Lib}$ and the Be star $\chi$ Oph in the region of the higher Paschen lines. The weak absorption distorting the blue wing of P15 in $48 \mathrm{Lib}$ is Ca II $\lambda 8542 \AA$. Ordinate is in units of intensity normalized to the continuum. Original dispersion: $23 \AA \mathrm{mm}^{-1}$.

\subsection{Ca II $(\lambda 8498 \AA, 8542 \AA$, and $8662 \AA)$}

The calcium triplet was found in emission in less than $20 \%$ of the objects surveyed. This is a lower percentage than has been reported by the earlier investigators. The probable reason for this is illustrated in Figure 4. All three calcium lines are severely blended with Paschen lines: Ca II $\lambda$ 8498, 8542, and $8662 \AA$ are respectively $4.5,3.3$, and $3.9 \AA$ blueward from P16, P15, and P13. In stars with strong Paschen emission or a strong Paschen shell, apparent weak $\mathrm{Ca}$ II emission is seen on lower dispersion plates (our $34 \AA \mathrm{mm}^{-1}$ plates and those of the early investigators). However, higher dispersion $\left(23 \AA \mathrm{mm}^{-1}\right)$ plates taken at the same time do not confirm the emission; they indicate only Paschen emission.

The presence of $\mathrm{Ca}$ II triplet emission in Be stars presents an interesting paradox. The lines arise from the ${ }^{2} P$ to ${ }^{2} D$ transition. The upper level $\left({ }^{2} P\right)$ is the same as for the $\mathrm{H}$ and $\mathrm{K}$ lines; the lower $\left({ }^{2} D\right)$ level is strongly metastable. The triplet lines are very subordinate transitions to the $\mathrm{H}$ and $\mathrm{K}$ lines, however, in no case do we observe 


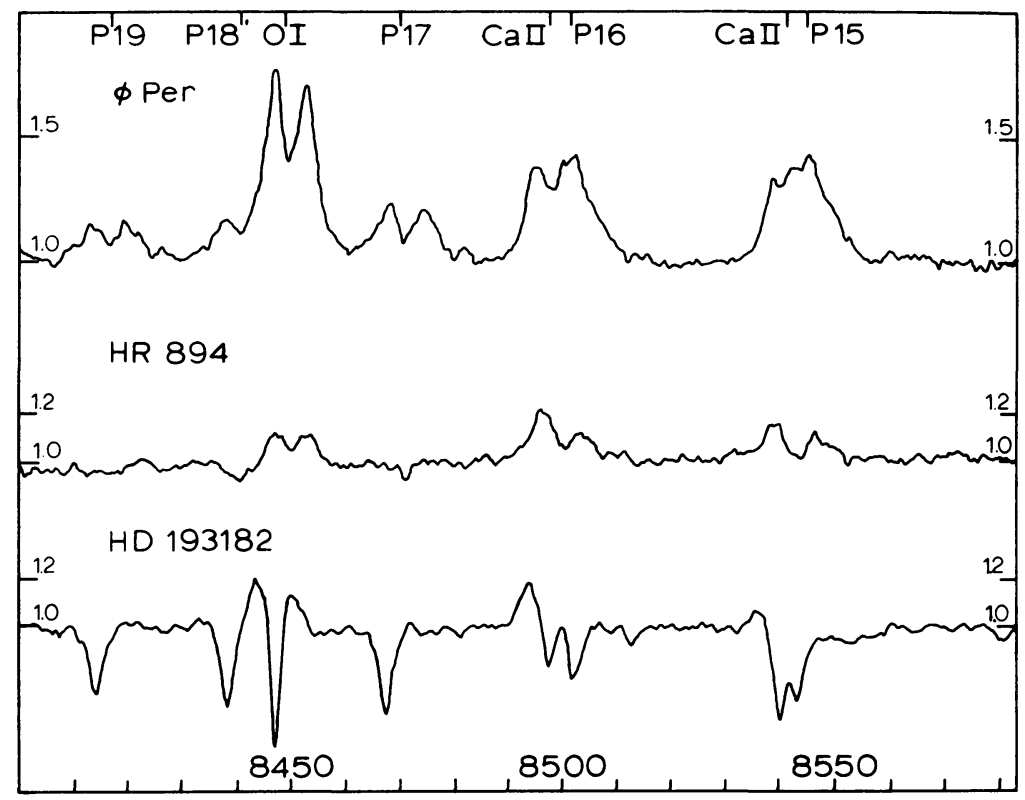

Fig. 4. Intensity tracings of the region of the higher Paschen lines in the Be stars $\phi$ Per and HR 984 and the shell star HD 193182. Note the strong emission at the Ca II lines $\lambda 8498 \AA$ and $\lambda 8542 \AA$. Ordinate and original dispersion same as in Figure 3.

significant $\mathrm{H}$ and $\mathrm{K}$ emission in a Be star showing the triplet in emission. The triplet lines themselves do not appear normal: all have the same intensities rather than the expected $1: 9: 5$. This implies that the gas giving rise to the emission is optically thick to the triplet. This, combined with the greater flux at $\lambda 3900 \AA$ than $\lambda 8500 \AA$, reduces the problem of the lack of $\mathrm{H}$ and $\mathrm{K}$ emission. It, however, does not eliminate it. One still requires a reduction of $\mathrm{H}$ and $\mathrm{K}$ emission with respect to the triplet of by a factor of at least 2 to 10. Calcium triplet emission also does not correlate with spectral type, emission envelope strength, the presence of dust or cool gas (as inferred from the far infrared flux), nor does it participate in variations seen in the rest of the envelope.

The best explanation for the presence of calcium triplet is to invoke the existence of dense cool $(T \sim 5000 \mathrm{~K})$ gas not associated with the visible envelope. The source of this gas is as yet unknown. However, the existence of visible cool $\left(T_{e} \sim 4-5000 \mathrm{~K}\right)$ giant companions to five of the objects showing triplet emission plus the presence of binary-like periodic activity in most of the other triplet emission objects suggest some connection with binary nature. A possible explanation is that the Be stars showing $\mathrm{Ca}$ II triplet emission are actually semi-detached interacting binary stars. The source of the cool gas is the mass losing contact component. A full discussion of the $\mathrm{Ca}$ II emission in Be stars along with its binary implications will be presented later in this symposium.

The lower level of the infrared calcium triplet is a strongly metastable level. In stars with extended envelopes one would expect to see these lines as strong shell lines. This is not seen. Calcium triplet shell lines are seen only in the cooler shell stars (e.g. 
$14 \mathrm{Com}, 1 \mathrm{Del})$ or the most extreme shell stars (e.g. $48 \mathrm{Lib})$. It is possible to understand this in terms of photoionization from the metastable level by the far ultraviolet photons $(\lambda \lesssim 1219 \AA)$. The metastable level has a large photoionization cross section, hence, no build up of atoms in this level is expected except in the stars with insufficient ultraviolet flux for photoionization or stars with very extensive envelopes.

\section{Be Binary Stars}

One of the primary purposes of this survey was to investigate the possibility that some Be stars are intereacting binary stars with cool companions. Two such systems are already known: 17 Lep and AX Mon. While it turns out that this region, $\lambda 8300$ to $8800 \AA$, is far from the optimum place to look for secondaries (this too will be discussed later in the Symposium), we would like to announce the discovery of two more Be binary stars. The well known periodic Be/shell star HD 218393 (Doazan and Peton, 1970) was found to contain an early K-type giant secondary (Figure 5).

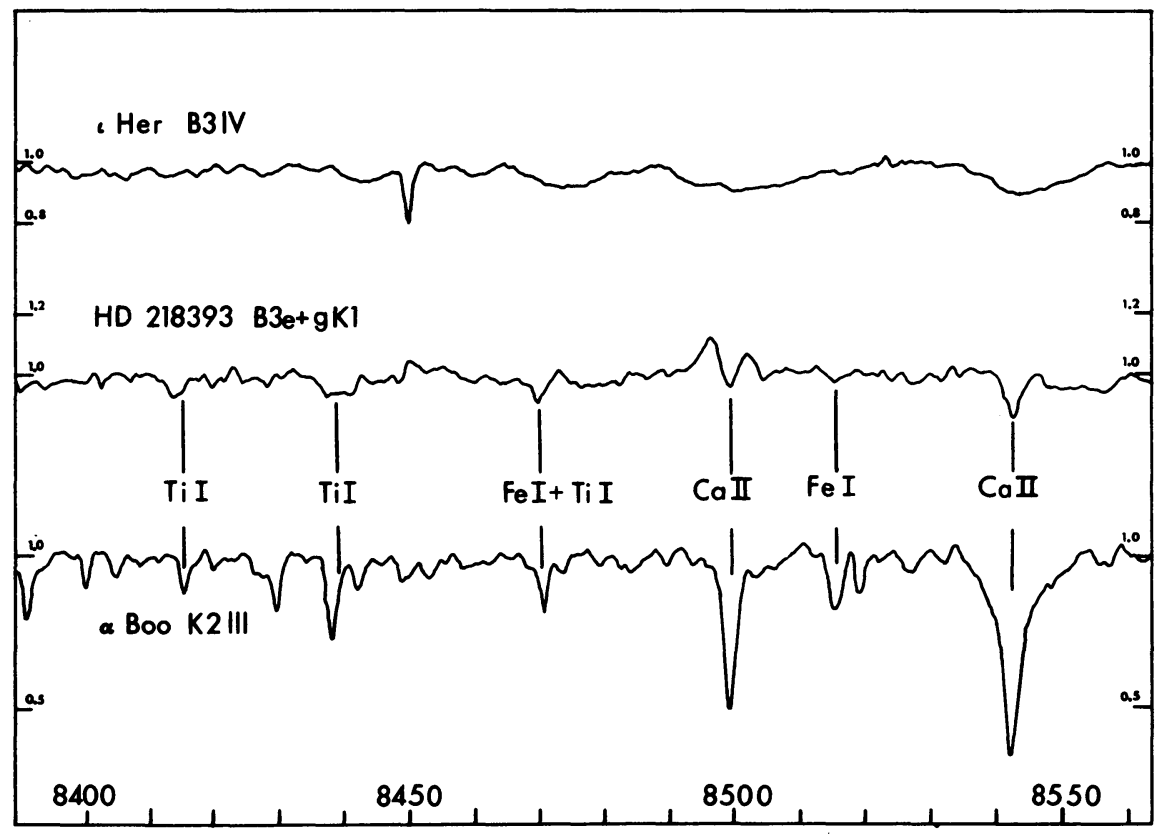

Fig. 5. The infrared spectrum of HD 218393 compared with that of $\iota$ Her (B3IV) and $\alpha$ Boo (K2III). The most prominent lines of the companion are marked. Ordinate and original dispersion same as for Figure 3.

The late B-type (B8V) emission star HR 894 (=HD 18552) was also found to contain a cool, in this case approximately G9, companion (Figure 6). Both these systems will be discussed in detail and compared to AX Mon, 17 Lep and other possible Be binary stars later in this Symposium. 


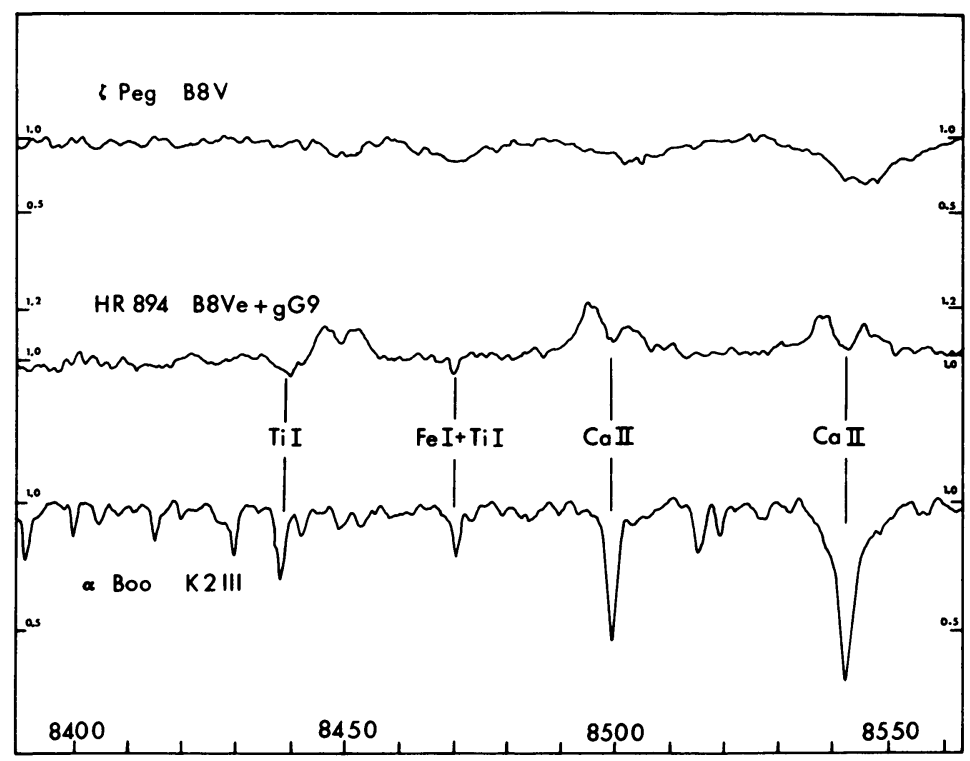

Fig. 6. The infrared spectrum of HR 894 compared with that of $\zeta \mathrm{Peg}$ (B8V) and $\alpha$ Boo (K2III). The most prominent lines of the companion are marked. Ordinate and original dispersion same as for Figure 3.

\section{Acknowledgements}

We wish to thank Dr M. Plavec for helpful discussion and for his continued support of the project. We also wish to thank Mr E. A. Harlan of Lick Observatory for assistance in obtaining some of the observations. This research was supported by NSF grant MPS 74-04194A01 (Popper/Plavec), being a part of a project studying close binary stars.

\section{References}

Andrillat, Y. and Houziaux, L.: 1967, J. Obs. 50, 107.

Andrillat, Y. and Houziaux, L.: 1972, Astrophys. Space Sci. 15, 240.

Bowen, I. S.: 1947, Publ. Astron. Soc. Pacific 59, 196.

Doazan, V. and Peton, A.: 1970, Astron. Astrophys. 9, 245.

Hiltner, W. A.: 1947, Astrophys. J. 105, 212.

Kitchin, C. R.: 1970, Monthly Notices Roy. Astron. Soc. 150, 455.

Kitchin, C. R. and Meadows, A. J.: 1970, Astrophys. Space Sci. 8, 463.

Osmer, P. S.: 1972, Astrophys. J. Suppl. Ser. 24, 247.

Slettebak, A.: 1951, Astrophys. J. 113, 436.

Zappala, R. R.: 1971, 'Proc. Conf. Late Type Stars', Contr. Kitt Peak National Obs. No. 554, 1. 